\title{
The molecular mechanisms of inhibitory effect of androstenone on hepatic skatole metabolism in relation to boar taint
}

E. Doran ${ }^{1}$, J. D. McGivan 2 , F. M. Whittington ${ }^{1}$ and J. D. Wood ${ }^{1}$

${ }^{I}$ Department of Clinical Veterinary Science, University of Bristol, Langford, Bristol BS40 5DU, U.K.

${ }^{2}$ Department of Biochemistry, University of Bristol, University Walk, Bristol BS8 1TK, U.K.

E-mail: E.Udovikova@bristol.ac.uk

Introduction Boar taint is off-odours in cooked pork from uncastrated male pigs. It is caused by an excessive accumulation of skatole and androstenone in backfat. Accumulation of skatole is due to a low expression and activity of hepatic enzyme CYP2E1. The mechanism of androstenone accumulation is not clear. It could be due to low activity and expression of 3 $\beta$-hydroxysteroid dehydrogenase (HSD), an enzyme metabolising androstenone in liver. On the basis of our previous in vivo experiments with castrated animals we suggest that accumulation of skatole is regulated by androstenone. Castrated pigs manifest lower levels of skatole and androstenone and higher CYP2E1 expression. We hypothesise that high levels of androstenone inhibits CYP2E1 expression and hence, reduces the rate of hepatic skatole metabolism. The aims of the present study were (i) to investigate the expression of androstenone-metabolising enzyme HSD in liver of pigs with high and low skatole and androstenone deposition; (ii) to investigate the effect of androstenone on expression of the skatole-metabolising enzyme CYP2E1 in vitro (in cell culture).

Materials and methods Uncastrated Large White x Landrace (LW) and Meishan x Landrace (M) male pigs, which are known as low and high skatole depositors respectively, were used in the study (five animals per each experimental group). The pigs were fed a standard pelleted diet and slaughtered at the carcass weight 60-70 kg. Skatole and androstenone levels in backfat were measured by high resolution gas chromatography. The rate of androstenone metabolism was estimated by measuring the formation of $\beta$-androstenol (using HRGC) after incubation of isolated microsomes with $1 \mathrm{mM}$ androstenone and $1 \mathrm{mM}$ NADH. CYP2E1 protein expression was analysed by Western Blotting with commercial antibodies. HSD cDNA level was measured by competitive PCR. Effect of androstenone on CYP2E1 expression was studied using cultured pig hepatocytes. The significance of differences was assessed by Student's $t$-test.

Results The rates of androstenone metabolism (Fig 1) and the amount of HSD cDNA (which reflects the HSD expression) (Fig 2), were higher in LW pigs when compared to M. The level of androstenone in backfat followed an opposite pattern: it was higher in M pigs and lower in LW (Fig.3). This indicates that high androstenone deposition is due to low expression of the androstenone metabolising enzyme. In cultured pig hepatocytes, skatole activated CYP2E1 expression and this activition was inhibited by androstenone (Fig.4). The results confirm our hypothesis that skatole accumulation is a result of repression of CYP2E1 expression by high levels of androstenone.

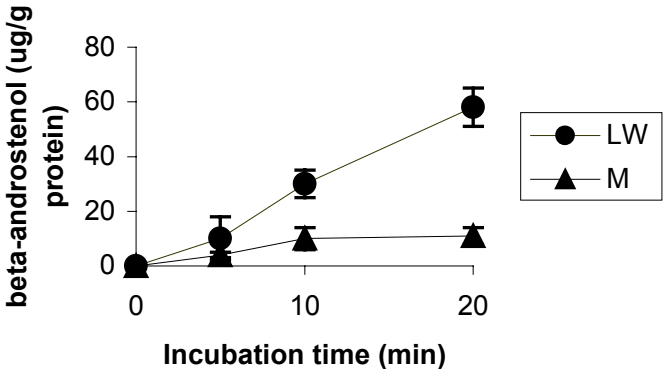

Figure 1 Rate of androstenone metabolism

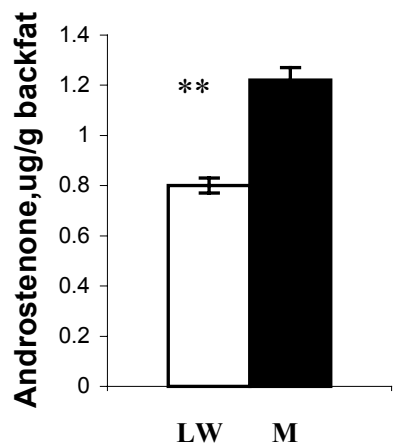

Figure 3 Androstenone level in backfat $* * \mathrm{P}<0.01$

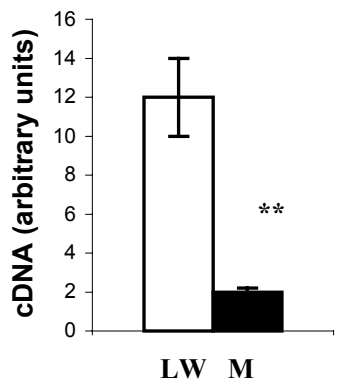

Figure 2 HSD cDNA in liver, $* * \mathrm{P}<0.01$

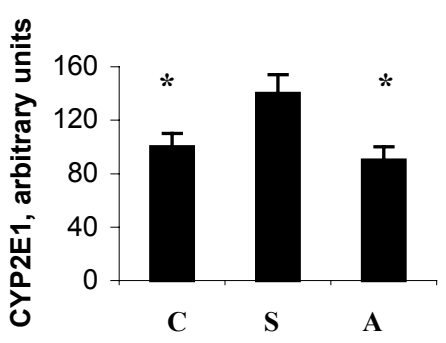

Figure 4 Effect of androstenone on CYP2E1 protein expression: $\mathrm{C}=$ control, $\mathrm{S}=$ plus $0.5 \mathrm{mM}$ skatole, $\mathrm{A}=$ plus $1 \mathrm{mM}$ androstenone $* \mathrm{P}<0.05$ when compared to $\mathrm{S}$

Conclusions Excessive accumulation of backfat skatole might be secondary to the accumulation of androstenone: a high level of androstenone inhibits expression of the skatole metabolising enzyme CYP2E1. An excessive accumulation of androstenone in backfat is due to low activity and expression of hepatic HSD. HSD can be considered as a possible physiological candidate gene for boar taint. 